\title{
Aporte de nutrientes em diferentes compartimentos de Eucalipto no sul do Tocantins
}

\author{
Maria Cristina Bueno Coelho ${ }^{\mathrm{a} *}{ }^{\circledR}$, Eziele Nathane Lima $^{\mathrm{a}}$, Mauro Luiz Erpen ${ }^{\mathrm{b}}{ }^{\oplus}$, \\ Juliana Barillia ${ }^{\mathrm{a}}$, Maurilio Antônio Varavallo ${ }^{\mathrm{a}}$, Marcos Vinicius Cardoso Silva ${ }^{\mathrm{a}}{ }^{\circledR}, \operatorname{Marcos}_{\text {Giongo }}{ }^{\mathrm{a}}$
}

a Universidade Federal do Tocantins, Brasil
b Instituto Federal do Tocantins, Brasil
*Autor correspondente (mariacristina@uft.edu.br)

\section{N F O}

\section{Keywords}

nutrient cycling

soil

bark

leaf

litter

\begin{abstract}
A B S T R A C T
Supply of nutrients in different compartments of Eucalyptus in southern Tocantins. The understanding of the nutrient cycling mechanisms of a forest plantation will allow the adequate management to harvest the wood or other products without any future damage to the soil. Therefore, the objective this work was to evaluate the nutritional content in the soil, litterfall, leaf and bark compartments in commercial plantation at age 7 with two clones: VM 58 from the crossing of Eucalyptus camadulensis $\mathrm{x}$ E. urophylla, the hybrid GG100 clone obtained from the crossing of E. urophylla x E. grandis, hybrids seeds of E. urophylla $\mathrm{x}$. grandis in the Aliança, Tocantins. The total biomass of $\mathrm{N}, \mathrm{P}, \mathrm{K}, \mathrm{Ca}, \mathrm{Mg}, \mathrm{S}, \mathrm{B}$ and the $\mathrm{Cu}, \mathrm{Fe}, \mathrm{Mn}$ and $\mathrm{Zn}$ micronutrients was estimated by estimating total aerial biomass using allometric equations. The $E$. urophylla x E. grandi svia seminal presented higher aerial biomass with 24,08 t.ha ${ }^{-1}$, and the lowest biomass yield was obtained by clone VM 58 with 13,09 t.ha ${ }^{-1}$. The average representativeness in decreasing order for aerial biomass for both eucalyptus species was: stem $(55 \%)>$ litterfall $(29 \%)>$ leaves (16\%). The soil obtained higher values for K and Fe but didn't differ in both nutrients. The leaves had higher concentrations for $\mathrm{N}$ and $\mathrm{Mn}$, while in the litterfall only the macronutrients were highlighted.
\end{abstract}

\section{R E S U M O}

A cultura da mandioca apresenta alta relevância socioeconômica para o Brasil. Entretanto, na região oeste a compreensão dos mecanismos da ciclagem de nutrientes de um plantio florestal permite o manejo adequado para colheita da madeira ou outros produtos sem que haja danos futuros ao solo, por isso, este estudo objetivou avaliar o conteúdo nutricional nos compartimentos solo, serrapilheira, folha e casca em plantio de Eucalyptus spp. no sul do Estado do Tocantins com idade de 7 anos, sendo os clones: VM 58 (cruzamento de E. camadulensis x E. urophylla) e o clone GG100 (cruzamento do E. urophylla x E. grandis). Também foram avaliadas áreas a partir de sementes híbridas de E. urophylla x E. grandis todas no município de Aliança do Tocantins. Foram analisados os macronutrientes ( N, P, K, Ca, Mg, S, B ) e os micronutrientes ( $\mathrm{Cu}, \mathrm{Fe}, \mathrm{Mn}$ e $\mathrm{Zn}$ ) e estimando a biomassa aérea total por meio de equações alométricas. O E. urophylla x E. grandis via seminal apresentou maior biomassa aérea com 24,08 t.ha- ${ }^{-1}$, e o menor rendimento de biomassa foi obtido pelo clone VM 58 com 13,09 t.ha-1. A representatividade média em ordem decrescente para biomassa aérea para ambos as espécies de eucaliptos foi: fuste (55\%) >serrapilheira $(29 \%)>$ folhas $(16 \%)$. O solo obteve maiores valores para $\mathrm{K}$ e Fe porém não diferiu em ambos os nutrientes. As folhas obtiveram maior concentração para $\mathrm{N}$ e Mn, enquanto na serrapilheira somente os macronutrientes destacaram. 


\section{INTRODUÇÃO}

Schumacher (1996) diz que no ecossistema florestal, a capacidade produtiva do sítio florestal será mantida por um período variado de acordo com a eficácia na reposição dos nutrientes através da utilização da biomassa, devido à perda de nutrientes por erosões. Por isso, faz-se necessário quantificar a biomassa produzida e a quantidade de nutrientes exportadas em cada compartimento como tronco, folhas, serrapilheira e solo. A compreensão dos mecanismos da ciclagem de nutrientes de um plantio florestal permitirá a exploração, para que ocorra o manejo adequado durante a colheita da madeira (De Carvalho et al., 2015).

A maioria das espécies de Eucalyptus spp., é originária da Austrália, tendo sido introduzida no Brasil por volta de 1904 adaptando-se bem às condições edafoclimáticas, hoje a cultura atende essencialmente o mercado de papel de celulose, mas também atende a indústria de carvão, mourões, óleos essenciais, entre outras (Angeli, 2004). Alguns gêneros da mesma espécie apresentam boa adaptação da árvore em eficiência nutricional superior às demais espécies, em particular as coníferas, devido à maior capacidade de translocação de nutrientes (Schumacher et al., 2011). Apresentando potencial de adaptação em regiões de déficit hídrico, as espécies desse gênero permitem sua implantação no cerrado, pois os solos dessa região, na grande maioria, apresentam baixa fertilidade e déficit hídrico em certa estação do ano (Carneiro et al., 2009).

Solos de regiões tropicais como os solos do cerrado geralmente são ácidos e com baixa fertilidade, apresentando alto potencial de lixiviação de nutrientes, principalmente o nitrogênio $(\mathrm{N})$ e potássio $(\mathrm{K})$ (Gama-Rodrigues et al., 2008). E para produção de biomassa são necessárias grandes quantidades de nutrientes, por isso, em um ecossistema florestal o solo não é apenas um meio de produção, mas um componente central mediador do processo solo-planta (Carneiro et al., 2009). Também os compartimentos folhas e galhos finos são componentes que permanecem na área em forma de resíduos após a colheita sendo grandes influenciadores da fertilidade na camada superficial do solo. Segundo Ferreira (1984), em um estudo com E. Grandis, concluiu que em média $80 \%$ do fósforo (P) e do $\mathrm{N}$ que retornam ao solo são oriundos das folhas.

No entanto, a camada formada pela serrapilheira em conjunto com o sistema radicular e a parte aérea das plantas protege o solo de agentes erosivos que também possibilita melhores condições para restabelecimento das propriedades físicas, químicas e biológicas (IKPE et al., 2003). Logo, a dinâmica dos nutrientes da serrapilheira ocorre pela entrada via deposição de todo material e sua saída via decomposição, tal processo é importante para manutenção dos plantios florestais (Lima et al., 2015).

Desta forma a ciclagem de nutrientes está diretamente ligada com o manejo eficiente do povoamento florestal, com as diferentes quantidades de nutrientes contidas na parte aérea, raízes, serrapilheira e solo, que contribuem para o fluxo contínuo entre o que está sendo depositado no solo e o que está sendo assimilado pelas raízes.

Tendo em vista a relevância da utilização de Eucalyptus spp para auxiliar no manejo florestal, este trabalho teve como objetivos analisar os atributos físicos e químicos do solo e estimar o estoque de nutrientes nos diferentes componentes da biomassa.

\section{MATERIAL E MÉTODOS}

\section{Área de estudos}

Os dados foram coletados no ano de 2018 no município de Aliança, Estado do Tocantins localizado nas coordenadas geográficas a latitude $11^{\circ} 18^{\prime} 22^{\prime \prime}$ e longitude $48^{\circ} 56^{\prime} 09^{\prime \prime}$ com elevação de $257 \mathrm{~m}$ e temperatura média anual de $26^{\circ} \mathrm{C}$.

$\mathrm{O}$ clima, segundo Thornathwaite consiste em C2wA'a" (SEPLAN, 2012), clima úmido, subúmido com moderada deficiência hídrica no inverno, evapotranspiração potencial média anual de $1.500 \mathrm{~mm}$, distribuindo-se no verão em torno de $420 \mathrm{~mm}$ ao longo dos três meses consecutivos sendo junho, julho e agosto com temperatura mais elevada.

A área tinha plantio de 7 anos de idade com os clones VM 58 (cruzamento de E. camaldulensis X E. urophylla), com 47,93 ha, o clone GG100 (cruzamento do E. urophylla x E. grandis ), correspondendo a 35,65 ha e 25 ha plantados com sementes híbridas de $\mathrm{E}$ urophylla $\mathrm{x} \mathrm{E}$ grandis.

Foi realizada uma adubação de base incorporada três meses antes do plantio feito com subsolador, aplicando-se o fosfato natural no fundo do sulco. Em seguida, foram realizadas duas adubações de cobertura com NPK (20-5-20) super simples; a primeira cobertura foi realizada aos 3 meses após o plantio e a segunda foi realizada nove meses após o plantio. E por fim, realizou-se uma adubação de manutenção (NPK) com 25 meses após o plantio. Em relação aos tratos culturais foram feitos limpeza mecanizada, combate manual as formigas e cupins e replantio feito 30 dias após o plantio.

O solo foi caracterizado como Latossolo vermelho-amarelo distrófico com relevo plano suave ondulado. Estes ocorrem em ambientes bem drenados, sendo muito profundos e uniformes em 
características de cor, textura e estrutura em profundidade (análise de solo feita no laboratório de solo da UFT campus de Gurupi).

Para a coleta da casca, realizou-se uma cubagem rigorosa pelo método de Smalian de 10 árvores (duas árvores de diâmetro médio; duas árvores de diâmetro médio mais um desvio-padrão, duas árvores de diâmetro médio menos um desviopadrão, duas árvores de diâmetro médio mais dois desvios padrão e duas árvores de diâmetro médio menos dois desvio padrão) por cada espécie de Eucalyptus sp, num total de 30 árvores feita a partir de inventário florestal sistemático e estratificado a partir de parcelas fixas de $27,5 \times 28 \mathrm{~m}\left(770 \mathrm{~m}^{2}\right)$ totalizando 42 parcelas (14 parcelas por espécie) onde foram determinadas as $\mathrm{Ht}$ (altura total medida em metros com hipsômetro) e DAP (diâmetro a altura do peito medido em $\mathrm{cm}$ com sutta) sendo que as amostras de casca foram retiradas com as seguintes alturas: de $0,5 \mathrm{~m} ; 1,0 \mathrm{~m} ; 1,3 \mathrm{~m}$ e de $2 \mathrm{~m}$ em $2 \mathrm{~m}$ até a extremidade da árvore de menor espessura com circunferência igual a $15,7 \mathrm{~cm}(5$ $\mathrm{cm})$ que foram pesadas e colocadas para secar ao ar livre por 48 horas e posteriormente levadas à estufa a $70^{\circ} \mathrm{C}$ por mais 72 horas.

Para a análise foliar foram coletadas amostras de ramos situados nas posições base, meio e topo das copas, segundo as quatro direções dos pontos cardeais (Oliveira et al., 2001).

As amostras de solo superficiais (profundidades de $0-20 ; 20-40$ e 40 a $60 \mathrm{~cm}$ ) foram obtidas a partir de 3 amostras compostas (total de 45 amostras simples por clone, ou seja, a cada 15 subamostras foi feito uma amostra composta em cada profundidade) por clone e via seminal a partir da metodologia da EMBRAPA (Filizola et al., 2006).

A coleta de serrapilheira seguiu o protocolo da EMBRAPA (Arevalo et al., 2002) para estimativa da biomassa florestal e carbono orgânico, coletada com auxílio de gabarito metálico (dimensões de 0,5 x $0,5 \mathrm{~m}$ ), lançado de forma aleatória em cada área (30 amostras por área num total de 90). As memas foram acondicionadas em sacos de papel, pesadas em balança digital e posteriormente levadas ao laborartório para serem secas em estufa a $70{ }^{\circ} \mathrm{C}$ por 48 horas.
As amostras de biomassa foram secas a $70^{\circ} \mathrm{C} \mathrm{em}$ estufa de circulação e renovação de ar, até a estabilização da massa, e pesadas em balança de precisão para determinação da massa seca. Após a secagem, as amostras foram moídas em moinho de lâminas do tipo Wiley com peneira de 20 mesh.O material coletado foi tratado e analisado para N, P, $\mathrm{K}, \mathrm{Ca}, \mathrm{Mg}, \mathrm{S}, \mathrm{B}, \mathrm{Cu}, \mathrm{Fe}, \mathrm{Mn}$ e $\mathrm{Zn}$ de acordo com as instruções contidas em Sarruge \& Haag (1974).

\section{Determinação da Biomassa Aérea}

A biomassa da serrapilheira foi determinada utilizando o peso seco da amostra coletada dividido pelo peso fresco da amostra, e por fim, utilizando o fator de correção $(0,04)$ para determinar em toneladas por hectare. Utilizou-se a seguinte equação:

$$
B A H\left(\frac{t}{h a}\right)=\left(\frac{P S M}{P F M}\right) * P F T * 0,04
$$

Em que:

BAH = biomassa arbustiva/herbácea, matéria seca; PSM = peso seco da amostra coletada; $\mathrm{PFM}=$ peso fresco da amostra coletada; $\mathrm{PFT}=$ peso fresco total por metro quadrado e fator de conversão $=0,04$.

A biomassa da casca e das folhas foram estimadas por equações alométricas simples e múltiplas (Tabela1), consequentemente, avaliando seus parâmetros estatísticos. Sendo testados 4 modelos com a utilização das variáveis dendrométricas altura (H) e (DAP). utilizando o software Excel 2013 e o Software SigmaPlot 10.0.

Após o ajuste dos modelos de regressão para a biomassa seca, verificou-se a qualidade do ajuste obtido por cada modelo pelo teste de KolmogorovSmirnov (K-S). Assim esse teste analisa com precisão as frequências estimadas através dos modelos de regressão testados com as frequências observadas.

Tabela1 - Modelos alométricos para determinação da biomassa aérea dos compartimentos casca e folhas.

\begin{tabular}{|c|c|}
\hline Número & Modelos \\
\hline 1 & $\operatorname{Ln} Y=\beta_{0}+\beta_{1} D A P^{-1}+\beta_{2} I^{-1}+\beta_{3}(D A P * I)^{-1}$ \\
\hline 2 & $Y=\beta_{0}+\beta_{1} D A P+\beta_{2} D A P^{2}+\beta_{3}(D A P * H)+\beta_{4}\left(D A P^{2} * H\right)+\beta_{5} H$ \\
\hline 3 & $Y=\beta_{0}+\beta_{1} D A P+\beta_{2} D A P^{2}+\beta_{3} D A P^{3}+\beta_{4} D A P^{4}$ \\
\hline & $Y=\beta_{0}+\beta_{1} D A P+\beta_{2} D A P^{2}+\beta_{3} D A P^{0}+\beta_{4} D A P^{4}+\beta_{5} D A P^{5}$ \\
\hline
\end{tabular}

$\mathrm{Y}=$ biomassa estimada Ln: logaritmo neperiano; $\beta_{0}, \beta_{1}, \beta_{2}, \beta_{3}, \beta_{4}$ e $\beta_{5}$ : coeficientes dos modelos; DAP: diâmetro da altura do peito; H: altura total; I: idade. 
O teste de K-S basicamente compara a frequência acumulativa estimada com a frequência observada. O maior ponto de divergência é o valor de D entre duas distribuições pelo teste de K-S.

$$
D_{\text {calc }}=\frac{S U P_{X}\left|F o_{(x)}-F e_{(x)}\right|}{n}
$$

Em que:

$\mathrm{F}_{\mathrm{o}(\mathrm{x})}$ : frequência observada acumulada; $\mathrm{F}_{\mathrm{e}(\mathrm{x})}$ : frequência esperada acumulada; $\mathrm{n}$ : Número de observações; $\mathrm{D}_{\text {calc }}$ : Valor $\mathrm{D}$ calculado.

\section{Análise estatística dos dados}

Com base no inventário florestal, foi determinada a estatística descritiva para o diâmetro e a biomassa seca foi obtida baseado no teor de umidade das amostras, e o conteúdo de nutrientes, pelo produto entre a biomassa seca e o teor de nutrientes, em cada componente das árvoresamostra. O estoque de nutrientes por unidade de área foi estimado pelo produto entre o conteúdo médio de nutrientes em cada componente da biomassa das árvores-amostra e o número de árvores por hectare.

Para análise estatística foi utilizado o software Sisvar 5.6 (Ferreria, 2011) para realizar o teste Tukey a $\mathrm{p}<=0,05$ de significância, para determinar a diferença entre os macronutrientes e micronutrientes do solo, da casca, folha e serrapilheira. Realizou-se a análise de correlação linear de Pearson (r) calculado e o cálculo da biomassa aérea no Excel 2013, e por fim, os graficos de balanço de nutrientes foram gerados no software Sigmaplot 10.0. A seleção da melhor equação foi determinada pelo coeficiente de determinação ajustado e coeficiente de variação.

\section{RESULTADOS E DISCUSSÃO}

O solo foi classificado como textura média, mostrando que a área tem $\mathrm{pH}$ com acidez elevada $\mathrm{e}$ CTC baixa, teores elevados de $\mathrm{K}$, teores muito baixos de $\mathrm{P}$, logo, teores baixos de $\mathrm{Ca}$ e $\mathrm{Mg}$ (Ribeiro et al., 1999). Na tabela 2, observa-se a análise estatística pelo teste Tukey a 5\% de significância dos macronutrientes e micronutrientes em tha ${ }^{-1}$ das espécies E. urophylla $\mathrm{x}$ E. grandis via seminal, cloneVM58 e GG100 para solo.

Tabela 2 - Análise granulométrica e características químicas do solo das espécies de E. urophylla x E. grandis via seminal (VS), clone VM58 e GG100 na área de estudo.

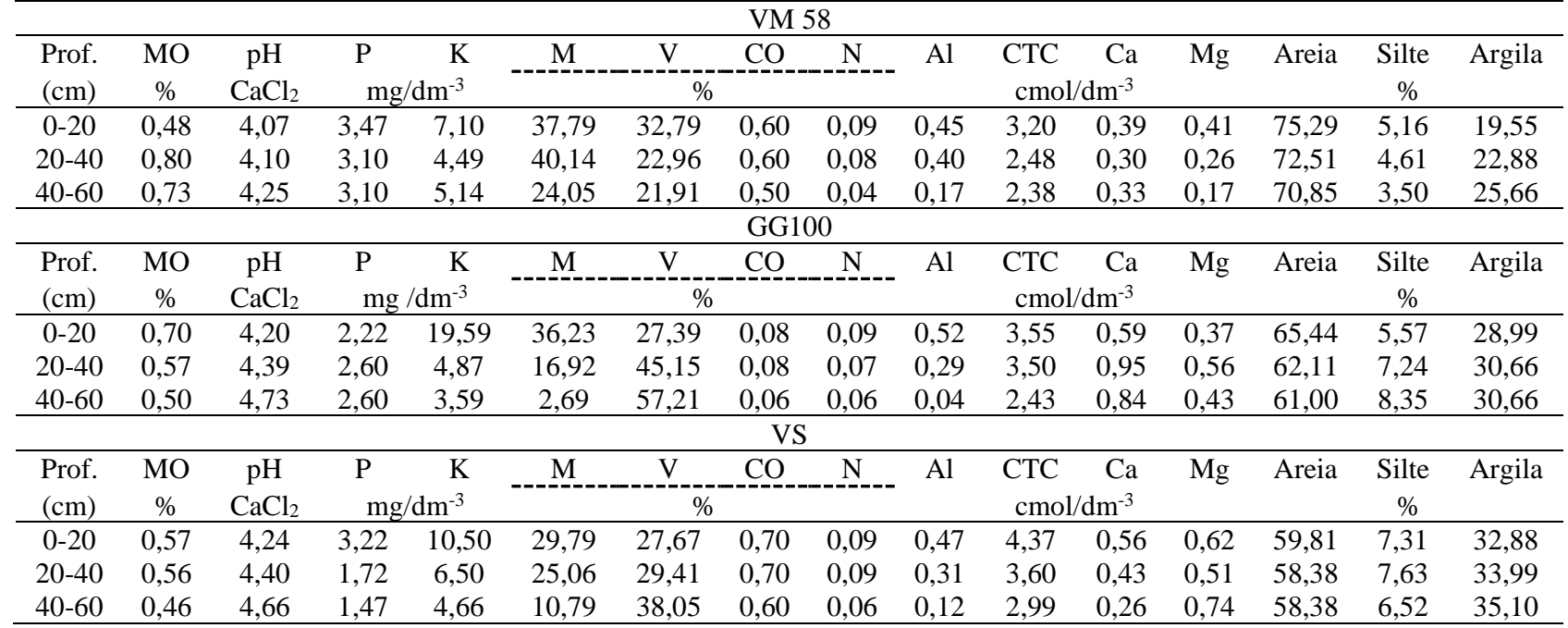

Em que: $\mathrm{MO}=$ matéria orgânica em percentagem; $\mathrm{pH} \mathrm{CaCl} 2$ = leitura do $\mathrm{pH}$ em cloreto de cálcio; $\mathrm{P}=$ fósforo $\mathrm{em} \mathrm{mg} / \mathrm{dm}^{-3} ; \mathrm{K}=$ potássio em $\mathrm{mg} / \mathrm{dm}^{-3} ; \mathrm{M}=$ saturação por alumínio em $\% ; \mathrm{V}=$ saturação pro base em $\% ; \mathrm{CO}$ = carbono orgânico em \%; $\mathrm{N}=$ teor de nitrogênio em $\mathrm{cmol} / \mathrm{dm}^{-3} ; \mathrm{Al}=$ teor de alumínio em $\mathrm{cmol} / \mathrm{dm}^{-3} ; \mathrm{CTC}=$ capacidade de troca catiônica em $\mathrm{cmol} / \mathrm{dm}^{-3} ; \mathrm{Ca}=$ teor de cálcio em $\mathrm{cmol} / \mathrm{dm}^{-3}, \mathrm{Mg}=$ teor de magnésio em $\mathrm{cmol} / \mathrm{dm}^{-3}$.

Segundo a Comissão de Química e Fertilidade do Solo - RS/SC (2004), o teor de matéria orgânica no solo é baixo ( $\leqslant 2,5)$; o pH é ácido, o $\mathrm{P}$ disponível são muito baixos $\left(\leqslant 5,0 ; \leqslant 3,0 \mathrm{mg} \mathrm{dm}^{-3}\right.$, respectivamente); o K trocável é baixo (40 - $120 \mathrm{mg}$ $\left.\mathrm{dm}^{-3}\right)$; o Ca e $\mathrm{Mg}$ trocáveis são baixos $(\leqslant 2,0 \mathrm{e} \leqslant$

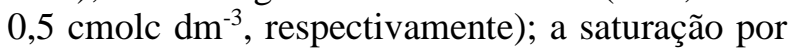
$\mathrm{Al}$ é alta (> 20\%) e a saturação por bases é de baixa 
a média $(\leqslant 20<45 \%)$. Com isso, de maneira geral, o solo da área experimental é de baixa fertilidade.

$\mathrm{O} \mathrm{N}$ do solo obteve o menor valor entre os macronutrientes, sendo explicado por Barreto et al. (2012), que os teores de $\mathrm{N}$ tendem a diminuir conforme a idade do povoamento que aumenta por unidade de biomassa, havendo uma eficiência quanto ao uso do $\mathrm{N}$ na produção de madeira, além de seu acréscimo na ciclagem biogeoquímica.

$\mathrm{O}$ Ca é o segundo elemento com maior taxa nutricional, de acordo com Schumacher et al. (2008), o Ca é devolvido ao solo via serrapilheira e absorvido de maiores profundidades, apresentando alto teor para os clones VM 58, no entanto, os valores para o clone GG 100 e via seminal foram semelhantes.

Os micronutrientes para os compartimentos casca apresentaram valores não significativos entre as espécies de Eucalyptus do trabalho (Tabela 3). O Fe apresentou os maiores valores das espécies entre os micronutrientes, pois quanto maior a concentração de Fe, menor a concentração de $\mathrm{P}$, seguindo a mesma relação decrescente para o clone VM 58 e o E. urophylla $x$ E. grandis: $\mathrm{Fe}>\mathrm{Cu}>\mathrm{Mn}>\mathrm{Zn}>\mathrm{B}$ e para clone GG 100 ficou a sequência $\mathrm{Fe}>\mathrm{Mn}>$ $\mathrm{Cu}>\mathrm{Zn}>\mathrm{B}$.

Os estoques de nutrientes nos componentes da biomassa de Eucalyptus sp. nos povoamentos via seminal, clone VM 58 e o clone GG100, em valores absolutos, para macro e micronutrientes, respectivamente são apresentados na tabela 3. Considerando-se os valores médios, os principais componentes da biomassa arbórea em acúmulo de nutrientes foram: folha >casca> serrapilheira; enquanto os nutrientes, apresentaram a seguinte ordem $\mathrm{N}>$ $\mathrm{Ca}>\mathrm{K}>\mathrm{Mg}>\mathrm{P}$. A distribuição de nutrientes geralmente difere do padrão observado para a biomassa, o que decorre da grande diferença na concentração de nutrientes entre os componentes (Valente et al., 2016).

Tabela 3 - Teores de nutrientes nos compartimentos casca, folha e serrapilheira das espécies de E. urophylla $x$ E. grandis via seminal, clone VM58 e GG100 em t.ha ${ }^{-1}$.

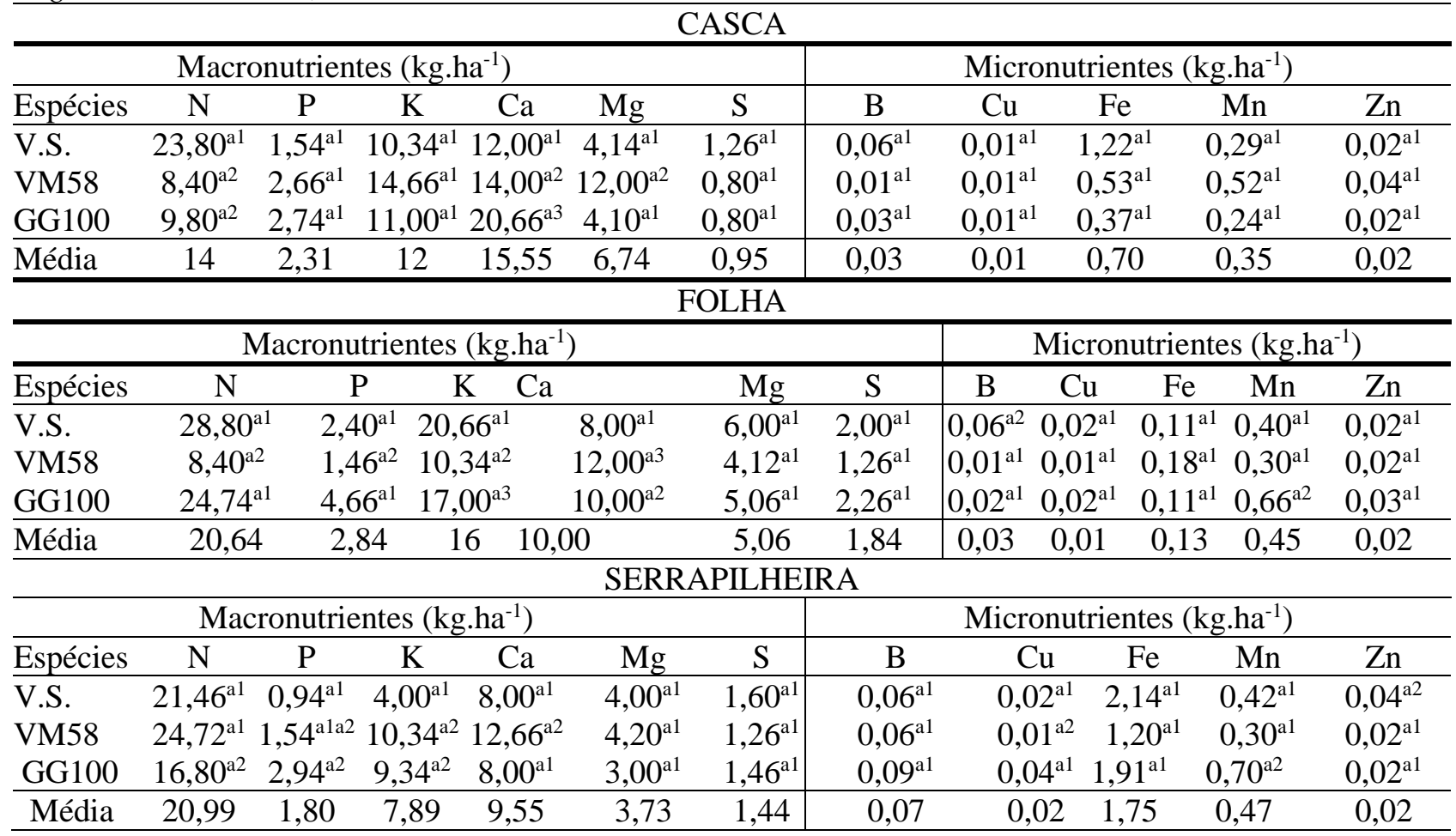

*As médias seguidas pela mesma letra da coluna não diferem de acordo com o teste de Tukey, ao nível de 5\% de probabilidade.

Para o compartimento casca, os macronutrientes apresentaram diferença significativa entre as espécies de clones VM58 e GG100 para Ca com 14,0 e 20,6 t.ha ${ }^{-1}$, sendo o nutriente que mais acumula neste compartimento, por isso é importante que durante a colheita as árvores sejam descascadas na área, garantido a economia de nutrientes para o solo.
O Mg diferiu apenas para o clone VM 58 obtendo 12,0 t.ha $^{-1}$, em uma pesquisa realizada com E. camaldulensis com 6 anos de idade encontrou maiores teores de $\mathrm{Ca}$ e $\mathrm{Mg}$ para o componente casca (Zaia e Gama-Rodrigues, 2004). O N, onde encontrou a maior taxa nutricional pode ser observado no clone GG100 com 23,80 t.ha ${ }^{-1}$. Então segue a seguinte relação: $\mathrm{N}>\mathrm{Ca}>\mathrm{K}>\mathrm{Mg}>\mathrm{P}>\mathrm{S}$. $\mathrm{Os}$ 
resultados do trabalho não foram os mesmos de acordo com Gatto et al. (2014), onde as relações da quantidade de nutrientes foram: $\mathrm{Ca}>\mathrm{K}>\mathrm{N}>\mathrm{Mg}$ $>\mathrm{P}>\mathrm{S}$, com os respectivos valores $0,0498>$ $0,03579>0,01668>0,00663>0,00165>0,00164$ t.ha $^{-1}$ em E. urophylla $\mathrm{x}$ E. grandis aos 5 anos. Os mesmos resultados não corroboraram com os de Vieira et al. (2013) em E. urophylla $x$ E. globulus de 48 meses.

Para os micronutrientes, a relação da quantidade dos nutrientes é: $\mathrm{Fe}>\mathrm{Mn}>\mathrm{B}>\mathrm{Zn}>\mathrm{Cu}$, salientando que na análise estatística não houve diferença significativa entre os micronutrientes (Tabela 3). Já para Guimaraes et al. (2015), os resultados não foram semelhantes com o presente trabalho, com a seguinte quantidade de nutrientes: $\mathrm{Mn}>\mathrm{Fe}>\mathrm{B}>\mathrm{Zn}$ $>\mathrm{Cu}$ em Eucalyptus dunnii de 48 meses

Para o clone VM 58, houve uma diferença significativa no $\mathrm{P}$ e Ca, com 1,46 e 12 t.ha ${ }^{-1}$, respectivamente, onde o maior valor é observado no $\mathrm{Ca}$ e na mesma espécie, um menor valor para o $\mathrm{P}$, com a relação da quantidade de nutrientes: $\mathrm{N}>\mathrm{K}>\mathrm{Ca}$ $>\mathrm{Mg}>\mathrm{P}>\mathrm{S}$. Nos micronutrientes a única diferença significativa foi para o Mn, onde o clone GG100 obteve o maior valor com 0,66 th ha $^{-1}$, e a sua relação de nutrientes: $\mathrm{Mn}>\mathrm{Fe}>\mathrm{B}>\mathrm{Zn}>\mathrm{Cu}$.

De acordo com Scheer (2008), os macronutrientes $\mathrm{K}$ e $\mathrm{Mg}$ das folhas apresentam uma taxa com maior velocidade de ciclagem no ecossistema, onde esses foram depositados pela própria vegetação. Os resultados do trabalho não assemelharam com os de Matos et al. (2012) para as espécies de E. grandis X E. urophylla, E. grandis $\mathrm{x}$ E. pellita, E. grandis $\mathrm{x}$ E. Urophylla e E. camaldulensise aos 17 meses de idade.

Os macronutrientes obtiveram diferença significativa para $\mathrm{Pe} \mathrm{Ca}$, onde 2,94 t.ha ${ }^{-1}$ para o $\mathrm{P}$ no clone GG100 é o maior valor, e para Ca 12,66 t.ha ${ }^{-1}$ para o clone VM58, a quantidade por ordem decrescente: $\mathrm{N}>\mathrm{Ca}>\mathrm{K}>\mathrm{Mg}>\mathrm{S}>\mathrm{P}$ (Tabela 4). Já para os micronutrientes a diferença significativa é observada no $\mathrm{Cu}, \mathrm{Mn}$ e $\mathrm{Zn}$, e sua relação de quantidade em ordem decrescente: $\mathrm{Fe}>\mathrm{Mn}>\mathrm{B}>\mathrm{Zn}>\mathrm{Cu}$.

Viera et al. (2013) estudando o conteúdo de nutrientes na serrapilheira acumulada em E. grandis, encontrou valores médios para as concentrações de $\mathrm{K}$ e Ca bastante superiores aos encontrados no presente estudo, sendo: 10,46 e 19,73 g. $\mathrm{kg}^{-1}$, respectivamente. $\mathrm{O} \mathrm{Ca}$ apresentou o segundo maior teor entre os macronutrientes, explicado pela sua baixa mobilidade no tecido vegetal, sendo determinante para maior quantidade na ciclagem deste nutriente durante a queda das folhas e pela decomposição no solo.

De acordo com Alvarez et al. (2007), o S possui alta concentração na serrapilheira, principalmente em folhas mais velhas por estar nelas retido, sendo incorporado aos esqueletos carbônicos, Assim como o $\mathrm{Mg}$ e Ca, há uma capacidade de distribuição baixa, por se concentrarem em tecidos mais velhos, mas o trabalho não corroborou com a afirmação de Lima et al. (2011).

$\mathrm{O}$ micronutriente $\mathrm{B}$ foi o terceiro com maior valor encontrado no trabalho, onde este se assemelha com o trabalho de Pérez et al. (2013). O fato de o B ser o terceiro elemento com maior concentração na serrapilheira é devido a limitação de sua mobilidade (Caldeira et al., 2007) ou até mesmo a imobilidade deste elemento no tecido vegetal (Kirby \& Römheld, 2007), ocorrendo a contribuição do acúmulo do B em folhas mais velhas. No trabalho o $\mathrm{Zn}$ é o um dos elementos que apresenta menor concentração na serrapilheira, considerando o elemento com baixa mobilidade em relação ao B.

$\mathrm{O} \mathrm{Cu}$ foi o microelemento que ocorreu em menor concentração na serrapilheira em relação aos outros elementos, devido a ser considerado pouco móvel na planta. (Kirby e Römheld, 2007). É importante frisar que a concentração nutricional de cada indivíduo sofre alteração em função da idade, características nutricionais e nível de fertilidade do solo (Schumacher e Poggiani 1993).

Com base na análise de regressão, foram estimadas as equações com melhores ajustes e menores erros para a estimativa da biomassa por compartimento do Eucaliptus (Tabela 4). As equações obtidas com a análise de regressão apresentaram coeficiente de determinação ajustado acima de $39 \%$ e baixo erro padrão das estimativas variando de 0,42 a 29,85, tendo demonstrado, com isso, boa predição das variáveis analisadas. Com ajuste das equações, a maior biomassa aérea foi encontrada para o $E$. urophylla $x E$. grandis via seminal totalizando 24,806 t.ha $^{-1}$ e o menor rendimento de biomassa foi constatado no clone VM 58 com 13,09 t.ha ${ }^{1}$. A representatividade em ordem decrescente para biomassa aérea foi: fuste > serrapilheira> folhas.

De acordo com os resultados obtidos para o teste de normalidade de Kolmogorov-Smirnov, mostrouse que as equações são adequadas para estimar a biomassa aérea de fuste e folhas, aceitando a hipótese de normalidade $\left(\mathrm{H}_{0}\right)$, em que, os dados estimados se ajustaram aos modelos para determinação da biomassa dos compartimentos, onde os valores críticos bilaterais calculados a 5\% e 1\% de significância encontrados foram 0,409 e 0,489 para as três espécies de Eucalyptus: o E. urocan (VM 58), E. urograndis (GG 100) e E. urograndis via seminal, sendo que os valores tabelados foram iguais aos valores críticos calculados. 
Tabela 4 - Coeficientes das regressões e estatísticas dos compartimentos fuste e folhas para os clones VM 58, GG 100 e seminal.

\begin{tabular}{|c|c|c|c|c|c|c|c|c|c|c|c|}
\hline Clone & Comp & $\mathrm{N}^{\circ}$ & $\beta_{0}$ & $\beta_{1}$ & $\beta_{2}$ & $\beta_{3}$ & $\beta_{4}$ & $\beta_{5}$ & $\mathrm{CV}$ & $\mathrm{Raj}^{2}$ & Syx $\%$ \\
\hline \multirow{8}{*}{$\sum_{>}^{\infty}$} & \multirow{4}{*}{ 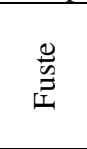 } & 1 & 5,064 & $-14,955$ & & & & \multirow{4}{*}{$-1276,438$} & 3,40 & 0,11 & 0,42 \\
\hline & & 2 & 32280,347 & $-4263,183$ & 138,395 & 168,900 & $-5,481$ & & 33,8 & 0,74 & 19,72 \\
\hline & & 3 & $-68096,137$ & 18237,368 & $-1815,101$ & 79,606 & $-1,298$ & & 24,6 & 0,39 & 26,89 \\
\hline & & 4 & $-586795,470$ & 191671,870 & $-24917,381$ & 1611,937 & $-51,904$ & & 24,9 & 0,40 & 29,85 \\
\hline & \multirow{4}{*}{$\begin{array}{l}\text { है } \\
\stackrel{7}{0} \\
\text { i }\end{array}$} & 1 & 6,860 & $-52,784$ & & & & \multirow{3}{*}{304,190} & 14,22 & 0,71 & 0,34 \\
\hline & & 2 & $-7781,552$ & 991,542 & $-30,803$ & $-38,848$ & 1,215 & & 45,30 & 0,67 & 17,66 \\
\hline & & 3 & 84012,019 & $-22249,333$ & 2194,728 & $-95,554$ & 1,549 & & 44,45 & 0,64 & 16,37 \\
\hline & & 4 & $-238855,744$ & 85706,097 & $-12185,435$ & 858,255 & $-29,950$ & 0,414 & 44,64 & 0,65 & 18,16 \\
\hline \multirow{8}{*}{$\frac{8}{\circlearrowright}$} & \multirow{4}{*}{ 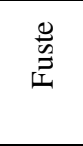 } & 1 & 7,734 & $-52,343$ & & & & \multirow{3}{*}{208,551} & 18,39 & 0,82 & 0,36 \\
\hline & & 2 & $-4197,628$ & 606,017 & $-20,887$ & $-30,359$ & 1,071 & & 58,37 & 0,85 & 23,27 \\
\hline & & 3 & $-8276,551$ & 2369,059 & $-250,366$ & 11,584 & $-0,196$ & & 58,11 & 0,85 & 21,34 \\
\hline & & 4 & 371740,015 & $-142788,859$ & 2174 & -164 & 61,398 & $-0,910$ & 59,43 & 0,88 & 20,63 \\
\hline & \multirow{4}{*}{ 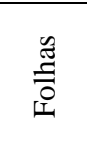 } & 1 & 2,326 & 11,653 & & & & \multirow{3}{*}{10,532} & 5,05 & 0,04 & 0,81 \\
\hline & & 2 & $-398,090$ & 71,491 & $-3,376$ & $-1,868$ & 0,101 & & 33,86 & 0,64 & 10,75 \\
\hline & & 3 & $-22442,444$ & 6814,523 & $-769,088$ & 38,283 & $-0,709$ & & 38,31 & 0,82 & 6,76 \\
\hline & & 4 & 56026,916 & $-23159,035$ & 3773,666 & $-303,102$ & 12,009 & $-0,187$ & 38,75 & 0,84 & 7,14 \\
\hline \multirow{8}{*}{ 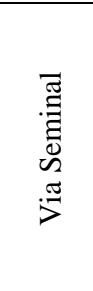 } & \multirow{4}{*}{$\begin{array}{l}\frac{0}{0} \\
\frac{0}{I}\end{array}$} & 1 & 7,370 & $-40,693$ & & & & \multirow{3}{*}{223,045} & 12,29 & 0,78 & 0,31 \\
\hline & & 2 & $-5791,911$ & 840,545 & $-29,769$ & $-32,462$ & 1,172 & & 52,32 & 0,91 & 23,98 \\
\hline & & 3 & 5998,898 & $-1800,329$ & 198,284 & $-9,455$ & 0,166 & & 49,83 & 0,83 & 30,34 \\
\hline & & 4 & $-104764,718$ & 36291,880 & $-4996,026$ & 341,627 & $-11,596$ & 0,156 & 50,04 & 0,84 & 33,21 \\
\hline & \multirow{4}{*}{$\begin{array}{l}\stackrel{0}{7} \\
\stackrel{0}{0} \\
\text { II }\end{array}$} & 1 & 5,636 & $-30,344$ & & & & \multirow{3}{*}{$-32,486$} & 11,79 & 0,54 & 0,40 \\
\hline & & 2 & 506,365 & $-60,751$ & 1,912 & 4,137 & $-0,127$ & & 37,11 & 0,50 & 20,18 \\
\hline & & 3 & 18556,181 & $-5153,334$ & 529,182 & $-23,786$ & 0,395 & & 42,37 & 0,65 & 15,04 \\
\hline & & 4 & 116599,885 & $-38871,097$ & 5126,987 & $-334,551$ & 10,807 & $-0,138$ & 43,84 & 0,70 & 15,66 \\
\hline
\end{tabular}

Em que: $\beta_{0}, \beta_{1}, \beta_{2}, \beta_{3}, \beta_{4}$ e $\beta_{5}$ : Coeficientes dos modelos; CV: coeficiente de variação (\%); Raj²: coeficiente de determinação ajustado; Syx: erro padrão da estimativa (\%).

Paes et al. (2013) na pesquisa realizada sobre a estimativa da biomassa de E. globulus encontrou um valor para biomassa aérea do fuste de 57.576 kg.ha-1 seguido das folhas com $10.539 \mathrm{~kg} \cdot \mathrm{ha}^{-1} \mathrm{e}$ ressalta que a distribuição da biomassa varia de acordo com a espécie, idade, sítio e condições ambientais. Neste trabalho, encontrou inferiores para fuste e folhas nos clones avaliados (Figura 1). Em trabalho realizado por Vieira et al. (2012) sobre biomassa e nutrientes em povoamento de $E$. urograndis com 18 meses na Serra do Sudeste-RS encontrou para fuste $6.843,7$ e folhas $3.943 \mathrm{~kg} \cdot \mathrm{ha}^{-1}$ biomassa seca da parte aérea, sendo resultados inferiores ao mostrando acima, podendo ser explicado pela idade do plantio estudado ser de 84 meses.

O E. urophylla $x$ E. grandis via seminal destacou-se entre os demais clones obtendo maior biomassa aérea e melhor teor nutricional para ambos os compartimentos, isso pode ser explicado pela qualidade do sítio e o tipo do material genético.

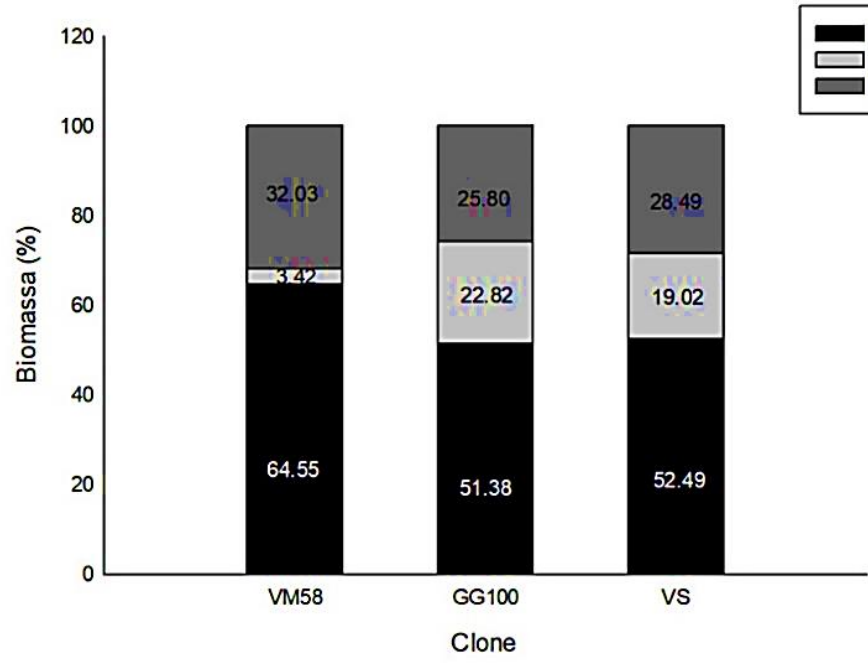

Figura 1 - Percentual de biomassa aérea por compartimento e por clone analisado. 
Tal comportamento é explicado pelo avanço da idade, os plantios clonais estabilizam o crescimento em altura e diâmetro, por serem geneticamente iguais. Essa característica possibilita ter maior controle na qualidade da madeira para o produto final, controla a incidência de pragas e doenças, enquanto os plantios por sementes continuam crescendo, tendo maior variabilidade, obtendo árvores heterogêneas e algumas com maior produção.

Vale ressaltar que, a produção de biomassa florestal é influenciada pela distribuição de chuvas na região, logo interfere na disponibilidade de água no solo, e também na disponibilidade de nutrientes das plantas, consequentemente, melhora o transporte de nutrientes do solo para as plantas e aumentando a biomassa (Selle et al., 2010).

Se considerarmos os valores médios para os povoamentos com 7 anos de idade, a serapilheira acumulada representou $42 \%$ do $\mathrm{Ca}, 22,85 \%$ do $\mathrm{Mg}, 44 \%$ do N, 5,58\% do P e 27,91\% do K, estocados na casca do tronco (Tabela 5). Verificase que os estoques na serapilheira são mais expressivos para nutrientes com baixa mobilidade no tecido vegetal. De acordo com Valente et al. (2016), a ciclagem interna é um importante processo para a manutenção dos nutrientes no interior da planta, e é mais importante para nutrientes como $\mathrm{N}, \mathrm{P}, \mathrm{K}$ e $\mathrm{Mg}$, e menos importante para $\mathrm{Ca}, \mathrm{S}$ e micronutrientes.

Tabela 5 - Valores percentuais de Macro e Micronutrientes por espécie e por compartimento.

\begin{tabular}{|c|c|c|c|c|c|c|c|c|c|c|c|}
\hline \multicolumn{7}{|c|}{ Macronutrientes } & \multicolumn{5}{|c|}{ Micronutrientes } \\
\hline \multicolumn{12}{|c|}{ Casca } \\
\hline Espécies & $\mathrm{N} \%$ & $\mathrm{P} \%$ & $\mathrm{~K} \%$ & $\mathrm{Ca} \%$ & $\mathrm{Mg} \%$ & $\mathrm{~S} \%$ & $\mathrm{~B} \%$ & $\mathrm{Cu} \%$ & $\mathrm{Fe} \%$ & $\mathrm{Mn} \%$ & $\mathrm{Zn} \%$ \\
\hline V.S. & 44.84 & 2.90 & 19.48 & 22.61 & 7.80 & 2.37 & 3.75 & 0.63 & 76.25 & 8.13 & 1.25 \\
\hline VM 58 & 15.99 & 5.06 & 27.91 & 26.66 & 22.85 & 1.52 & 0.90 & 0.90 & 47.75 & 46.85 & 3.60 \\
\hline GG 1 & 19.96 & 5.58 & 22.40 & 42.08 & 8.35 & 1.63 & 4.48 & 1.49 & 55.22 & 35.82 & 2.99 \\
\hline \multicolumn{12}{|c|}{ Folha } \\
\hline Espécies & $\mathrm{N} \%$ & $\mathrm{P} \%$ & $\mathrm{~K} \%$ & $\mathrm{Ca} \%$ & $\mathrm{Mg} \%$ & $\mathrm{~S} \%$ & $\mathrm{~B} \%$ & $\mathrm{Cu} \%$ & $\mathrm{Fe} \%$ & $\mathrm{Mn} \%$ & $\mathrm{Zn} \%$ \\
\hline V.S. & 42.44 & 3.54 & 30.45 & 11.79 & 8.84 & 2.95 & 9.84 & 3.28 & 18.03 & 65.57 & 3.28 \\
\hline VM 58 & 22.35 & 3.89 & 27.51 & 31.93 & 10.96 & 3.35 & 1.92 & 1.92 & 34.62 & 57.69 & 3.85 \\
\hline 00 & 38.83 & 7.31 & 26.68 & 15.69 & 7.94 & 3.55 & 2.38 & 2.38 & 13.10 & 78.57 & 3.57 \\
\hline \multicolumn{12}{|c|}{ errapilheira } \\
\hline Espécies & $\mathrm{N} \%$ & $\mathrm{P} \%$ & $\mathrm{~K} \%$ & $\mathrm{Ca} \%$ & $\mathrm{Mg} \%$ & $\mathrm{~S} \%$ & $\mathrm{~B} \%$ & $\mathrm{Cu} \%$ & $\mathrm{Fe} \%$ & $\mathrm{Mn} \%$ & $\mathrm{Zn} \%$ \\
\hline V.S. & 53.65 & 2.35 & 10.00 & 20.00 & 10.00 & 4.00 & 2.24 & 0.75 & 79.85 & 15.67 & 1.49 \\
\hline VM 58 & 45.18 & 2.81 & 18.90 & 23.14 & 7.68 & 2.30 & 3.77 & 0.63 & 75.47 & 18.87 & 1.26 \\
\hline GG 100 & 40.44 & 7.08 & 22.48 & 19.26 & 7.22 & 3.51 & 3.26 & 1.45 & 69.20 & 25.36 & 0.72 \\
\hline
\end{tabular}

Considerando-se os valores médios, os principais componentes da biomassa arbórea em acúmulo de nutrientes foram: folha > casca > serrapilheira; enquanto os macronutrientes, apresentaram a seguinte ordem $\mathrm{N}>\mathrm{Ca}>\mathrm{K}>\mathrm{Mg}>$ $\mathrm{P}>\mathrm{S}$ e para os micronutrientes a ordem foi: $\mathrm{Fe}>$ $\mathrm{Mn}>\mathrm{B}>\mathrm{Zn}>\mathrm{Cu}$. A distribuição de nutrientes geralmente difere do padrão observado para a biomassa, o que decorre da grande diferença na concentração de nutrientes entre os componentes (Valente et al., 2016). O principal exemplo disso são as folhas, consideradas o centro metabólico da planta, é o componente com a maior concentração para a maioria dos nutrientes (Medeiros et al., 2016), e geralmente, apresentam o menor acúmulo de biomassa, principalmente em povoamentos adultos.

Os macronutrientes e micronutrientes presentes em menores quantidades relativas na folha $e$ serrapilheira do tronco foram os mais abundantes na casca do tronco, o que é amplamente relatado na literatura científica para Eucalyptus spp (Dias et al., 2015; Witschoreck e Schumacher, 2015; Medeiros et al., 2016; Dick et al., 2016). A casca, por ter representado os maiores valores médios de biomassa nos povoamentos com 7 anos de idade, acumulou valores entre 22,61 a 42,08 \% do $\mathrm{Ca}, 7,8$ a $22,85 \%$ do $\mathrm{Mg}, 19,48$ a $22,4 \%$ do K, 2,9 a $5,58 \%$ do P e 15,99 a $44,84 \%$ do N.

A distribuição dos nutrientes nos componentes da árvore tem grande importância na nutrição de povoamentos florestais manejados em rotações sucessivas, pois o manejo intensivo pode aumentar significativamente a produção de biomassa, aumentando também a exportação de nutrientes do sítio (Vieira et al., 2014; Schumacher et al., 2013). A figura 2 representa o balanço nutricional para macronutrientes do sistema solo-folha-cascaserrapilheira ao serem retirados do solo no momento da colheita. Mostrando que, ambos os clones VM 58, GG 100 e o E. urophylla x E. grandis via seminal apresentariam déficit de $\mathrm{N}, \mathrm{P}$ e $\mathrm{K}$ ao retirar as cascas, folhas e serrapilheira do solo. 
Balanço nutricional solo $x$ planta - GG100

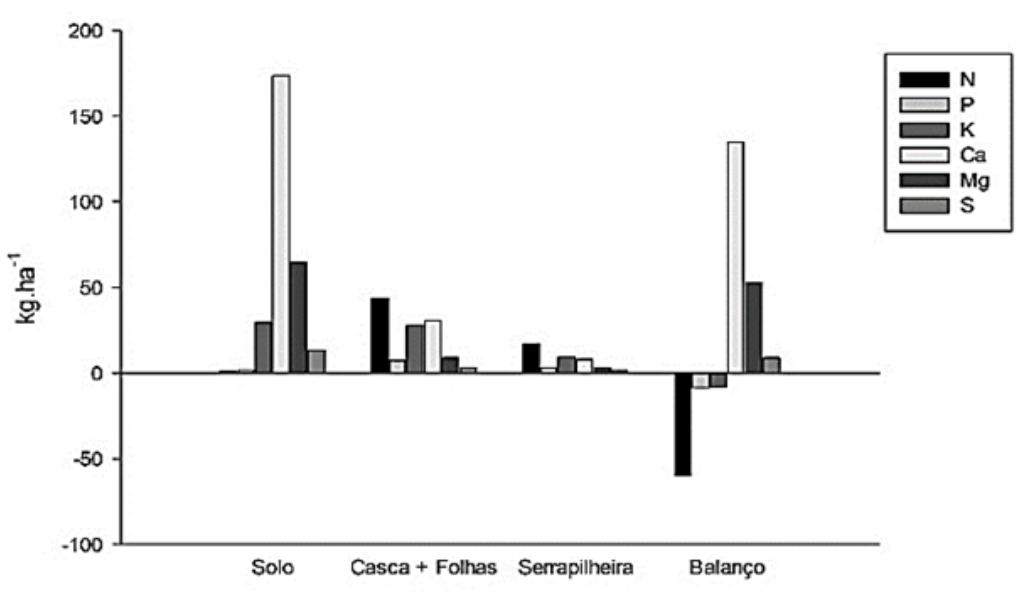

Balanço nutricional solo $\mathrm{x}$ planta - VM58

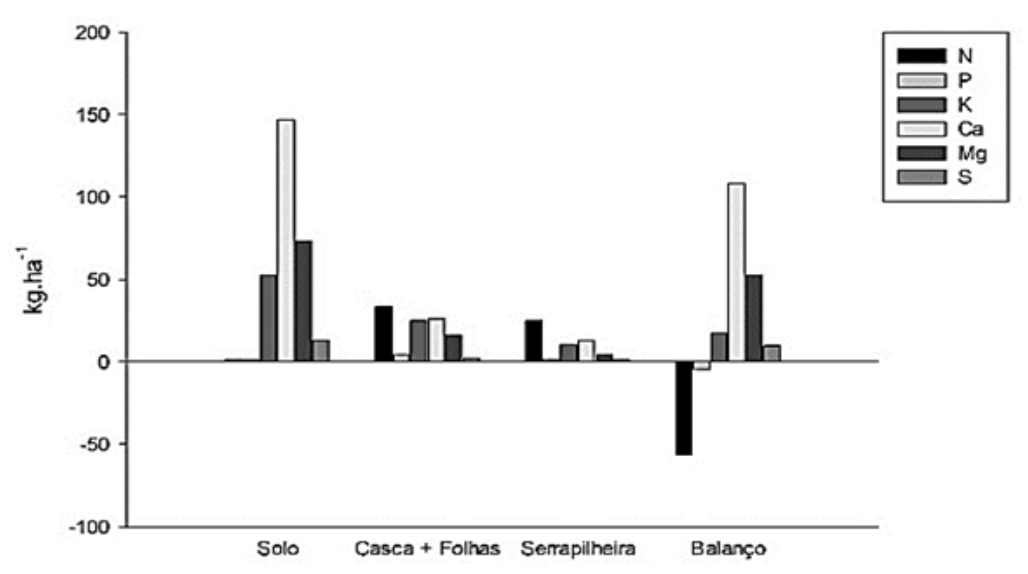

Balanço nutricional solo x planta - Via Seminal

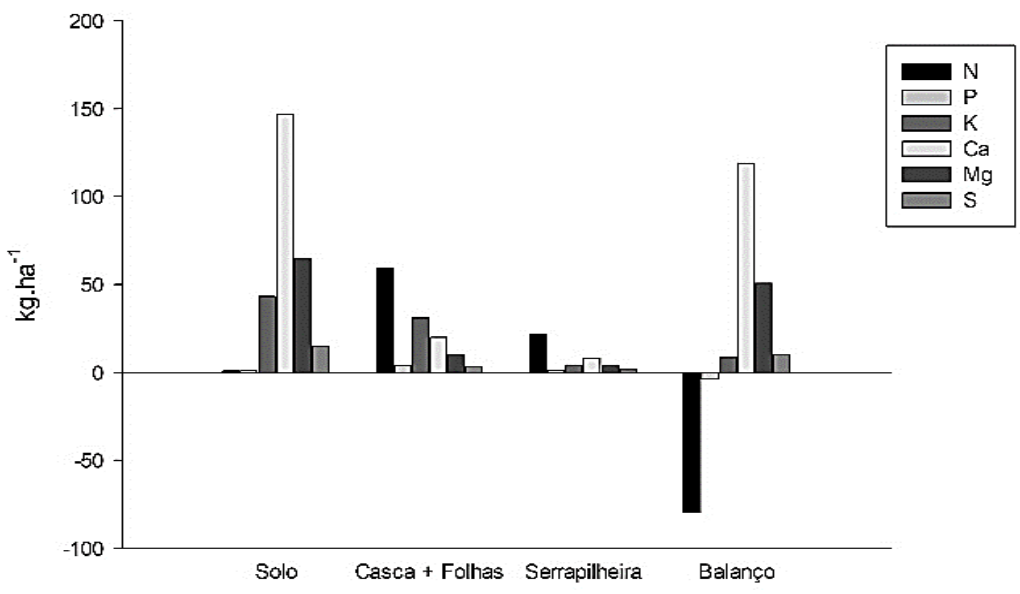

Figura 2: Balanco nutriconal para os macronutrientes do sistema solo, casca+folhas e serrapilheira para as diferentes espéices de Eucaliptus. 
Observa-se que, mesmo a fração folhas apresentando a menor biomassa, ela contém elevado estoque de nutrientes, como, por exemplo, de $\mathrm{N}\left(66 \mathrm{~kg}\right.$. ha $\left.{ }^{-1}\right)$. O balanço foi negativo para o $\mathrm{N}$, $\mathrm{K}$ e o $\mathrm{P}$ para o VM 58 e o GG100, devido a maior reserva de nutrientes encontrava-se no solo do que absorvido pela planta. No contexto geral, mesmo com saldo positivo para alguns nutrientes seu estoque não seria suficiente para suportar um novo ciclo florestal, necessitado de adubação.

Os demais nutrientes ficariam com saldo positivo no solo no balanço final, por isso, a importância do manejo correto dos plantios de Eucalyptus spp para que não esgotem o solo, mas que possa beneficiá-lo, para isso a necessidade de deixar os resíduos (galhos, folhas e casca) do plantio na área.

\section{CONCLUSÕES}

A quantidade de serrapilheira e nutrientes aportados demostra a importância desta via para ciclagem de nutrientes e para a manutenção da produtividade nas áreas florestais. Sendo que o maior acúmulo de nutrientes é no compartimento folhas que também é a responsável por maior parte do retorno de nutrientes via serapilheira.

$\mathrm{O}$ E. urophylla $x$ E. Grandis via seminal apresentou maior biomassa aérea com 24,08 t.ha${ }^{1}$, e o menor rendimento de biomassa foi obtido pelo clone VM 58 com 13,09 t.ha ${ }^{-1}$. A representatividade média em ordem decrescente para biomassa aérea para ambas as espécies de Eucalyptus foi: fuste $(55 \%)>$ serrapilheira $(29 \%)>$ folhas $(16 \%)$.

A biomassa aérea mostrou-se alocada principalmente na casca e também com os maiores estoques de nutrientes. apresentando composição química distinta, sendo geralmente maiores nas folhas e na serrapilheira.

No solo obteve-se os maiores valores para $\mathrm{K}$ e Fe porém, não houve diferença significativa para os nutrientes entre as espécies de Eucalyptus. Somente o Ca obteve maior valor diferindo dos demais nutrientes. O compartimento folha, obteve diferença significativa para os clones VM 58 e GG 100, destacando $\mathrm{P}$, Ca e $\mathrm{Mn}$, sendo que os observados com maior concentração de nutrientes foram o $\mathrm{N}$ e $\mathrm{Mn}$. Em relação a serrapilheira houve diferença significativa para o $\mathrm{P}, \mathrm{Ca}$ e $\mathrm{Cu}$ e obtevese maiores quantidades de nutrientes para $\mathrm{Ne} \mathrm{Fe}$.

A sequência para macronutrientes para os compartimentos folhas em ordem decrescente foi $\mathrm{N}>\mathrm{K}>\mathrm{Ca}>\mathrm{Mg}>\mathrm{P}>\mathrm{S}$, enquanto que para micronutrientes foi $\mathrm{Mn}>\mathrm{Fe}>\mathrm{B}>\mathrm{Zn}>\mathrm{Cu}$.

Os modelos lineares ajustados e testados nos 3 clones para os compartimentos fuste e folhas com até cinco coeficientes (todos com valores de $\mathrm{Raj}^{2}$ acima de $33 \%$ e valores menores de 20 para Syx\%) indicaram boa predição das variáveis analisadas e o teste de K-S indicou que os modelos selecionados são compatíveis para determinar a biomassa aérea

\section{REFERÊNCIAS BIBLIOGRÁFICAS}

Alvarez V, Roscoe VH, Roscoe R, Kurihara CH, Pereria NF. Enxofre. In: Novais et al. Ed. Fertilidade do Solo. Viçosa, MG: Sociedade Brasileira de Ciência do Solo, 2007. p.595644.

Angeli A. Indicações para escolha de espécies de Eucalyptus. IPEF - Instituto de Pesquisas e Estudos Florestais. 2004. Disponível em: Acesso em: julho. 2020.

Arevalo LA, Alegre JC, Montoya LJ. Metodologia para estimar o estoque de carbono em diferentes sistemas de uso da terra. Documento 73- Colombo: EMBRAPA Florestas, 2002.

Barreto PAB, Gama-Rodrigues AC, Gama-Rodrigues EF, Barros NF. Nitrogen balance in soilun de Eucalyptus plantations. Revista Brasileira Ciência do Solo, v.36, p.12391248, 2012.

Caldeira MVW, Marques R, Soares RV, Balbinot R. Quantificação de serrapilheira e de nutrientes - Floresta Ombrófila Mista Montana - Paraná. Revista Acadêmica, Curitiba, v.5, n.2, p.101-116, 2007. http://dx.doi.org/10.7213/cienciaanimal.v5i2.9720

Carneiro MAC, Edicarlos DS, Reis EF, Hamilton SP, Watson RA de. Atributos físicos, químicos e biológicos de Solo de cerrado sob diferentes sistemas de uso e manejo. Revista Brasileira de Ciência do Solo. Viçosa, v.33, n.1, p.147157, 2009. https://doi.org/10.1590/S010006832009000100016

Comissão de química e fertilidade do solo- RS/SC. Manual de adubação e calagem para os estados do Rio Grande do Sul e Santa Catarina. Porto Alegre, SBCS, 2004. 400p.

De Carvalho RR, Guimarães CC do, Silva JCM da, Momolli DR. Estoque de biomassa e de nutrientes em um povoamento do híbrido Eucalyptus urophylla $\mathrm{x}$ Eucalyptus grandis no Bioma Pampa-RS. Revista Ecologia e Nutrição Florestal-ENFLO, v.3, n.3, p.71-81, 2015. http://dx.doi.org/10.5902/2316980X18275

Dias LPR, Gatiboni LC, Brunetto G, Simonete BB. Eficiência relativa de fosfatos naturais na adubação de plantio de mudas de Eucalyptus dunnii Maiden e Eucalyptus benthamii Maiden et Cambage em solo sem e com calagem. Ciência Florestal, v.25, n.1, p.37-48, 2015.

http://dx.doi.org/10.1590/1980-509820152505037

Dick G, Momolli DR, Santos JC dos, Ludvichak AA, Guimarães CC, Souza, HP de. Quantificação da biomassa e nutrientes em um povoamento de Eucalyptus dunnii Maiden estabelecido no bioma Pampa. Ecologia e Nutrição Florestal, v.4, n.1, p.01-09, 2016.

http://dx.doi.org/10.5902/2316980X20589

Ferreira MGM. An Analysis of future productivity of Eucalyptus grandis plantation in Cerrado region in Brazil a nutrient cycling approach. Vancouver: University of Britsh Columbia. p.230.1984. 
Ferreira DF. Sisvar: A computer statistical analysis system.Ciência e Agrotecnologia, Lavras, v.35, n.6, p.10391042, nov./dez. 2011.

Filizola HF, Gomes MAF, Souza MD. Manual de procedimentos de coleta de amostras em áreas agrícolas para análise da qualidade ambiental: solo, água e sedimentos - Jaguariúna: EMBRAPA Meio ambiente, 2006.

Gama-Rodrigues AC, Gama-Rodrigues EF, Barros NF. Balanço de carbono e nutrientes em plantio puro e misto de espécies florestais nativas no sudeste da Bahia. Revista Brasileira de Ciência do Solo, v.32, n.3, p.1165-1179. junho, 2008.https://doi.org/10.1590/S010006832008000300025

Gatto A, Bussinguer AP, Ribeiro FC, Azevedo GB de, Bueno MC, Monterio MM, Souza PF de. Ciclagem e balanço de nutrientes no sistema de Eucalyptus sp., no Distrito Federal. Revista Brasileira de Ciência do Solo, v.38, p.879-887, 2014. https://doi.org/10.1590/S0100-06832014000300019

Guimarães CC, Schumacher MV, Witshoreck J, Souza HP, Santo JC. Biomassa e nutrientes em povoamentos de $E u$ calyptus dunnii Maiden no pampa gaúcho. Revista Árvore, Viçosa-MG, v.39, n.5, p.873-882, 2015. https://doi.org/10.1590/0100-67622015000500010

Ikpe FN, Owoeye LG, Gichuru MP. Nutrient recycling potential of Tephrosia candida in cropping systems of southeastern Nigeria. Nutrient Cycling in Agroecosystems, v.67, p.129-136, 2003. https://doi.org /10.1023/A:1025591719556

IPEF- Instituto De Pesquisa E Estudos Florestais. Pesquisa florestal no Brasil: Tecnologia de produtos florestais. Disponível em: http://www.ipef.br/mct/MCT_04.htm. Acesso em: 28 dez. 2018.

Kirkby EA, Römheld V. Micronutrientes na fisiologia de plantas: funções absorção e mobilidade. Informações Agronômicas, n.118, p.1-24, 2007.

Lima RLS de, Severino LS, Cazetta JO, Azevedo CAV. de.; Sofiatti V, Arriel NHC. Redistribuição de nutrientes em folhas de pinhão manso entre estádios fenológicos. Revista Brasileira de Engenharia Agrícola e Ambiental [online]., v.15, n.11. 2011. https://doi.org/10.1590/S141543662011001100010

Lima NL, Silva Neto CM, Calil FN, Souza R, de Moraes DC. Acúmulo de serapilheira em quatro tipos de vegetação no estado de Goiás. Enciclopédia Biosfera. v.11, n.22, p.3946. Goiânia-GO. 2015. https://doi.org/10.18677/EnciBio_2018A1

Matos GSB de, Silva GR, Gama MAP, Vale RS, Rocha EC da. Desenvolvimento inicial e estado nutricional de clones de eucalipto no nordeste do Pará. Revista Acta Amazonica. v.42, n.4, 2012, p.491-500. https://doi.org/10.1590/S004459672012000400006

Medeiros WN, Melo CAD, Tiburcio RAS, Silva GS da, Machado AFL, Santos LDT, Ferreira FA. Crescimento inicial e concentração de nutrientes em clones de Eucalyptus urophylla $\mathrm{x}$ Eucalyptus grandis sob interferência de plantas daninhas. Ciência Florestal, v.26, n.1, p.147-157, 2016. http://dx.doi.org/10.5902/1980509821099

Oliveira AC, Weiss D, Pinto LS, Reissmann CB. Procedimentos de coleta para análise foliar de essências florestais (araucária, erva-mate, palmito, eucalipto, pinus). Curitiba: Universidade Federal do Paraná, Projeto de Extensão Universitária Solo Planta, 2001. (Folder).

Paes FASV, Lima AMN, Hakamada NF de B, Barros NF. Effect of harvest residues management, soil tillage and fertilization on eucalyptus productivity. Revista Brasileira de Ciência do Solo, v.37, n.4, p.1081-1090, 2013. https://doi.org/10.1590/S0100-06832013000400025

Pérez C, Frangi JL, Goya JF, Luy A, Arturi MF. Contenido de nutrientes en las raíces finas y el mantillo de rodales de Eucalyptus grandis de diferente edad en la Mesopotamia Argentina. Bosque (Valdivia), v.34, n.3, p.303-310, 2013. https://doi.org/10.4067/S0717-92002013000300006

Ribeiro AC; Guimarães PTG, Alvarez VH. (ed.). Recomendação para o uso de corretivos e fertilizantes em Minas Gerais: 5a aproximação. Viçosa: CFSEMG, 359p. 1999.

Sarruge JR, Haag HP. Análises químicas em plantas. Piracicaba: Esalq. 1974

Scheer MB. Decomposição e Liberação de Nutrientes da Serrapilheira Foliar em um Trecho de Floresta Ombrófila Densa Aluvial em Regeneração, Guaraqueçaba (PR). Floresta, Curitiba, PR, v.38, n.2, abr./jun, 2008. http://dx.doi.org/10.5380/rf.v38i2.11620

Schumacher MV. Ciclagem de nutrientes como base da produção sustentada em ecossistema florestais. In: simpósio sobre ecossistemas naturais do Mercosul: o ambiente da floresta. Santa Maria. Anais. Santa Maria: UFSM-CEPEF, p.65-77. 1996

Schumacher MV, Witschoreck R, Calil FN. Biomassa em povoamentos de Eucalyptus spp. de pequenas propriedades rurais em vera cruz, RS. Ciência Florestal, Santa Maria, v.21, n.1, p.17-22, mar., 2011. http://dx.doi.org/10.5902/198050982743

Schumacher MV, Poggiani F. Produção de biomassa e remoção de nutrientes em povoamentos de Eucalyptus camaldulensis Dehnh, Eucalyptus grandis Hillex Maiden e Eucalyptus torelliana $\mathrm{F}$. Muell, plantados em Anhembi, SP. Ciência Florestal. v.3. p.21-34. 1993.

Schumacher MV, Vieira M, Witschoreck R. Produção de serrapilheira e transferência de nutrientes em área de segunda rotação com floresta de Pinus taeda L. no município de Cambará do Sul, RS. Ciências Florestais, Santa Maria, v.18, n.4, out.-dez., 2008. https://doi.org/10.5902/19805098431

Schumacher MV, Correa RS, Vieira M, Araujo EF de. Produção e decomposição de serapilheira em um povoamento de Eucalyptus urophylla $\mathrm{x}$ Eucalyptus globulus maidenii. Cerne, v. 19, n. 3, p. 501-508, 2013. https://doi.org/10.1590/S0104-77602013000300018

Selle GL. Ciclagem de nutrientes em ecossistemas florestais. Bioscience Journal. Uberlândia, v.23, n.4, p.29-39, out/dez 2010.

SEPLAN. Secretária do Planejamento e Orçamento (Seplan), 2012. Disponível em: www. Seplan.gov.br/ZEE/TO/atras_do_tocantins Acesso: $11 / 02 / 2019$.

Valente ML, Schumacher MV, Corrêa RS, Araújo EF, Trüby P. Quantification of nutrients in the rainfall in a Eucalyptus urophylla $\mathrm{x}$ Eucalyptus globulus subsp. maidenii 
plantation, Eldorado do Sul, RS. Scientia Forestalis, v.44, n.109, p.249-259, 2016. https://doi.org/10.18671/sci-

for.v44n 109.24

Vieira M, Backes KS, Kanieski MR, Chassot T, Souto L, Vieira M. Dinâmica nutricional em um povoamento híbrido de Eucalyptus urophylla x Eucalyptus globulus em Eldorado do Sul-RS, Brasil. Santa Maria, Universidade Federal de Santa Maria (Tese de Doutorado). 119p. 2012.

Vieira M, Scumacher MV, Trüby P, Araújo EF de. Biomassa e nutrientes em um povoamento de Eucalyptus urophylla $\mathrm{x}$ Eucalyptus globulus, em Eldorado do Sul-RS. Revista Ecologia e Nutrição Florestal-ENFLO, v.1, n.1, p.1-13, 2013. https://doi.org/10.1590/0100-67622015000500010

Vieira M, Scumacher MV, Araújo EF, Corrêa RS, Caldeira MVW. Deposição de serapilheira e nutrientes em plantio de Eucalyptus urophyllax E. globulus. Floresta e Ambiente, v.21, n.3, p.327-338, 2014.

https://doi.org/10.1590/2179-8087.053913

Witschoreck R, Schumacher MV. Nutrient allocation in Eucalyptus saligna $\mathrm{Sm}$. stands in the region of Guaíba-Rio Grande do Sul. Cerne, v.21, n.4, p.625-632, 2015. http://dx.doi.org/10.1590/01047760201521041963

Zaia FC, Gama-Rodrigues AC. Ciclagem e balanço de nutrientes em povoamentos de eucalipto na região Norte Fluminense. Revista Brasileira de Ciência do Solo. Viçosa, v.28, n.5. p.843-852. 2004. https://doi.org/10.1590/S010006832004000500007 